REVESCO. Revista de Estudios Cooperativos

ISSN: $1885-8031$

http://dx.doi.org/10.5209/REVE.58398

\title{
El marco legislativo y su efecto sobre el crecimiento del sector cooperativo en Colombia (1933-2014)
}

Giovanni Andrés Hernández Salazar ${ }^{1}$ y Ana María Olaya Pardo ${ }^{2}$

Recibido: 21 de octubre de 2015 / Aceptado: 29 de noviembre de 2017

Resumen. Esta investigación tiene como objetivo estimar cuantitativamente el efecto de las principales Leyes y Decretos promulgados para el sector cooperativo sobre el crecimiento del mismo en Colombia. La información se construye desde 1933-2014 a nivel Nacional y desde 1980-2014 a nivel departamental (regional). A partir de la estimación de modelos de intervención bajo la metodología ARIMA, se evidencia que el marco legal no he tenido un efecto estadísticamente significativo sobre el crecimiento del sector. Las principales razones que explican este resultado son la gran dispersión y falta de coherencia de la política cooperativa, el enfoque de la política más hacia la regulación, supervisión y vigilancia que al fomento y fortalecimiento, y a la insuficiente comprensión de los efectos negativos potenciales asociados a una estructura de propiedad colectiva.

Palabras clave: Cooperativas; ARIMA; Intervención; Política Pública.

Claves Econlit: P13; G38; L38.

\section{[en] Legislative framework and its effect on cooperative sector in Colombia (1933-2014)}

Abstract. This research aims to quantitatively estimate the effect of the main Laws and Decrees promulgated for the cooperative sector on its growth in Colombia. The information is constructed from 1933-2014 at the National level and from 1980-2014 at the departmental (regional) level. Based on the estimation of intervention models under the ARIMA methodology, it is evident that the legal framework has not had a statistically significant effect on the growth of the sector. The main reasons for this result are the wide dispersion and lack of coherence of the cooperative policy, the policy approach more towards regulation, supervision and surveillance than the promotion and strengthening, and insufficient understanding of the potential negative effects associated with a collective property structure.

Keywords: Cooperatives; ARIMA; Intervention; Public Policies.

Sumario. 1. Introducción. 2. Principales leyes cooperativas en Colombia. 3. Evolución del sector cooperativo en Colombia. 4. Metodología. 5. Discusión. 6. Conclusiones. 7. Recomendaciones. 8. Referencias bibliográficas.

1 Universidad de la Sabana, Colombia

Dirección de correo electrónico: giovannihs@unisabana.edu.co.

2 Universidad de la Sabana, Colombia

Dirección de correo electrónico: ana.olaya@unisabana.edu.co. 
Cómo citar: Hernández Salazar, G.A. y Olaya Pardo, A.M. (2018) El marco legislativo y su efecto sobre el crecimiento del sector cooperativo en Colombia (1933-2014). REVESCO. Revista de Estudios Cooperativos, Primer Cuatrimestre, No 127, pp. 139-158. DOI: 10.5209/REVE.58398.

\section{Introducción}

El desarrollo del sector cooperativo colombiano ha experimentado cuatro etapas importantes. Inicio y crecimiento (1933-1960) donde se institucionalizan las primeras cooperativas; fortalecimiento e integración (1961-1997) en la cual se incrementa el número de cooperativas constituidas y algunas se fusionan para conformar cooperativas de segundo grado; crisis (1998-1999) en la que instituciones financieras cooperativas fueron liquidadas, y post-crisis (2000 en adelante) donde emergen y fortalecen nuevas organizaciones.

El gobierno colombiano no es ajeno a esta historia. Por el contrario, ha promulgado un marco legislativo que busca el crecimiento, monitoreo y control del sector cooperativo. Esta investigación tiene como objetivo estimar cuantitativamente el efecto de las principales Leyes y Decretos sobre el crecimiento del sector cooperativo en Colombia, para el periodo 1933-2014 a nivel nacional y 1980-2014 a nivel regional (departamental).

La relevancia del estudio del sector cooperativo se evidencia por su importancia en la economía nacional. En el 2016 existían 3.666 cooperativas y 6.1 millones de asociados (13\% de la población total del país) que generaron cerca de 197.330 puestos de trabajo. Desde el punto de vista financiero, sus activos se estiman en $\$ 38.7$ billones de pesos, con un patrimonio de \$14.6 billones, pasivos de \$24.1 billones, ingresos de $\$ 27.9$ billones y excedentes por $\$ 748.000$ millones de pesos (CONFECOOP, 2016).

Los aportes de este documento a la comprensión del cooperativismo en Colombia son múltiples. Primero, se construye el registro del número de cooperativas desde 1933 hasta 2014 a nivel nacional y desde 1980 a 2014 a nivel departamental, series que no existían de forma continua en los archivos colombianos; segundo, se estima cuantitativamente el efecto de las principales Leyes y Decretos cooperativos sobre el crecimiento del sector en Colombia, y tercero, se identifican los aciertos y desaciertos de esta legislación a partir de la teoría de finanzas corporativas.

Este documento se organiza en cinco secciones: la primera, identifica las principales Leyes que rigen el sector cooperativo; la segunda, describe la evolución del sector cooperativo en Colombia, la tercera, muestra la metodología, los modelos estimados y resultados; la cuarta, presenta la discusión de los mismos. Finalmente, se exponen las conclusiones y recomendaciones.

\section{Principales leyes cooperativas en Colombia}

Desde al año 1931 hasta 2014 se sancionaron 15 Leyes y 28 Decretos para el sector cooperativo, 2 documentos de política de modernización y desarrollo del sector de la economía solidaria (CONPES 2823 de 1995 y 3639 de 2010), 1 Ley (Ley 454 de 1998) y 4 Decretos para la economía solidaria. Este entramado legal puede ser 
clasificado en Leyes y Decretos que buscan la formalización ${ }^{3}$, crecimiento $^{4}$, monitoreo y control ${ }^{5}$ del sector cooperativo, así como legislación especial para las cooperativas financieras de ahorro y crédito $^{6}$, y exenciones de impuestos ${ }^{7}$.

No obstante la copiosa legislación cooperativa, en "Colombia ha habido dos constituciones políticas (1886 y 1991) y tres leyes generales de cooperativas (ley 134 de 1931, decreto-ley 1598 de 1963 y ley 79 de 1988)... normas que han conformado la base de la normatividad aplicable al sector" (Procuraduría General de la Nación, 2011:70). Como complemento a esta legislación también se reconoce la importancia de la Ley 128 de 1936 (regula la creación de nuevas cooperativas), la Ley 24 de 1981 (por medio de la cual se crea el Departamento Administrativo Nacional de Cooperativas) y la Ley 454 de 1998 (constituye un marco conceptual

3 Es así que la Ley 134 de 1931 formaliza y define las sociedades cooperativas y el Decreto 4588 de 2006 define la organización y funcionamiento de las cooperativas y pre-cooperativas de trabajo asociado.

$4 \quad$ Para el crecimiento del sector cooperativo se sanciona la Ley 134 de 1931, el Decreto 874 de 1932 (declara las cooperativas como convenientes para la economía nacional), la Ley 128 de 1936 (normas para el fomento cooperativo), la Ley 61 de 1936, la Ley 115 de 1959 (cátedra de cooperativismo en instituciones educativas), la Ley 1598 de 1963 (segunda Ley General de Cooperativas), la Ley 1630 (fomento financiero), la Ley 79 de 1988 (tercera Ley General de Cooperativas), el Decreto 468 de 1990 (fomento a cooperativas de trabajo asociado), el Decreto 427 de 1996 (fácil constitución de cooperativas no financieras), el Decreto 2481 de 2003 (operaciones de redescuento por parte de FINDETER a las cooperativas).

5 El monitoreo y control se formaliza con el Decreto 1339 de 1932 (crea la Superintendencia de Sociedades Cooperativas), el Decreto 2392 de 1938 (pasa la Superintendencia de Cooperativas al Ministerio de trabajo), el Decreto 1649 de 1960 (establece el Consejo Nacional de Cooperativas), la Ley 1 de 1963 (actualización de legislación cooperativa), el Decreto Ley 1587 (se organiza la Superintendencia Nacional de Cooperativas), el Decreto Ley 1629 (estructuran funciones de la Superintendencia Nacional de Cooperativas), el Decreto 2059 de 1968 (Decreto reglamentario del Decreto Ley 1598 de 1963), la Ley 24 de 1981 (crea el Departamento Administrativo Nacional de Cooperativas -DANCOOP-), la Ley 454 de 1998 (crea la Superintendencia de Economía Solidaria -SuperSolidaria- y transforma el DANCOOP en el Departamento Administrativo Nacional de la Economía Solidaria -DANSOCIAL-), la Ley 365 de 1997 (control de lavado de activos del sistema financiero cooperativo), el Decreto 2206 de 1998 (crea el Fondo de Garantías de Entidades Cooperativas -FOGACOOP-), el Decreto 2506 de 1998 (Fondo de Solidaridad de Ahorradores y Depositantes de Entidades Cooperativas), el Decreto 2159 de 1999 (Niveles de Supervisión de la Entidades Vigiladas por la SuperSolidaria), el Decreto 727 (se realizan ajustes al FOGACOOP), el Decreto 1166 de 1999 (suprime el Departamento Administrativo Nacional de Economía Solidaria, el Consejo Nacional de Economía Solidaria y el Fondo de Fomento de la Economía Solidaria), el Decreto 1566 de 2000 (se adopta la estructura de DANSOCIAL y se señalan funciones), el Decreto 1153 (integración y funcionamiento del Consejo Nacional de la Economía Solidaria), el Decreto 186 de 2004 (se modifica la estructura de la SuperSolidaria), el Decreto 455 de 2004 (normas sobre la toma de posesión y liquidación de entidades vigiladas por la SuperSolidaria), el Decreto 2996 de 2004 (contenidos de estatutos de cooperativas de trabajo asociado), el Decreto 4672 de 2010 (crea la Comisión Intersectorial de Economía Solidaria), el Decreto 1712 de 2012 (reajusta el Consejo Nacional de Economía Solidaria).

6 Decreto 1111 de 1989 (actividades financieras), el Decreto 1134 de 1989 (actividades de ahorro y crédito), la Ley 45 de 1990 (intermediación financiera), el Decreto 663 de 1993 (cooperativismo financiero), la Ley 262 de 1996 (algunas cooperativas financieras pueden acceder a recursos del Fondo para el Financiamiento del Sector Agropecuario - FINAGRO-), el Decreto 1840 de 1997 (regulación prudencial de las cooperativas de ahorro y crédito), el Decreto 756 de 2000 (reglas para la toma de posesión de las cooperativas financieras), el Decreto 2886 de 2001 y 790 de 2003 (gestión de riesgo de liquidez) y el Decreto 795 de 2003 (disposiciones sobre regulación cooperativa financiera).

7 Ley 788 de 2002 (impuesto de renta y complementarios), el Decreto 2879 de 2004 (control de evasión y elusión de parafiscales de la cooperativas y empresas asociativas de trabajo), la Ley 1233 de 2008 (contribuciones a la seguridad social a cargo de las cooperativas y pre-cooperativas de trabajo asociado) y el Decreto 3553 de 2008 (reglamenta la Ley 1233 de 2008).

8 Ley 454 de 1998 abarca no solo el sector cooperativo sino todo el sector de la economía solidaria. Luis Razeto define la economía solidaria como "una búsqueda teórica y práctica de formas alternativas de hacer economía, basadas en la solidaridad y el trabajo" (Razeto, 2010). La cooperativa es una forma alternativa de hacer economía basada en la cooperación y solidaridad. La cooperativa es un ejemplo de organización que hace parte de la economía solidaria. 
que regula la economía solidaria). Estas Leyes establecen las normas generales y señalan los criterios y objetivos específicos establecidos en la Constitución (Artículo 150, numeral 19 Constitución Política de Colombia). Finalmente, son lo suficientemente generales para cobijar todas las organizaciones cooperativas sin importar el sector económico/social en la que se encuentran. Por estas razones este trabajo se concentra en el efecto de estas leyes sobre el fortalecimiento del sector cooperativo 9 .

\subsection{Ley 128 de 1936}

Esta Ley tiene como objetivo regular la creación de nuevas cooperativas y dictar normas en materia de impuestos. Obliga que la fundación de nuevas cooperativas sea autorizada por el poder ejecutivo y emite lineamientos sobre su constitución y obligaciones del gerente, tesorero y auditor. Establece de manera provisional los miembros del consejo de administración y la junta de vigilancia, y permite que estos sean ratificados previa aprobación por parte de la primera asamblea general.

Constituye alivios tributarios a través del no pago de impuestos de sellado, timbre, registro, anotación, patrimonio, fondos de reserva y solidaridad, acarreo, aduana, consular, tonelaje y puerto fluvial y publicaciones. Facilita los préstamos a las sociedades cooperativas con garantía de prenda industrial sobre los activos de las sociedades a través de la Caja de Crédito Agrario, Industrial y Minero. Crea el Consejo Nacional de Cooperación y expide actividades de vigilancia por parte del poder ejecutivo.

\subsection{Decreto Ley 1598 de 1963}

Fija la naturaleza y fines de la sociedad cooperativa como una organización de capital y asociados variables, con duración indefinida, funcionamiento democrático, igualdad de derechos y obligaciones entre los socios, con excedentes distribuidos a sus asociados acorde a las transacciones que cada uno realice con la cooperativa o según su participación en el trabajo, con un interés a capital no superior al 6\% anual y con el objetivo de fomentar la educación.

Presenta los lineamientos sobre constitución y reconocimiento, contenido de los estatutos, administración, funcionamiento y conformación de la asamblea general, consejo de administración, junta de vigilancia y comités especiales.

Reconoce a las sociedades cooperativas como personas jurídicas cuyas actividades deben ser de carácter social. Define las cooperativas especializadas como aquellas que solo se ocupan de una actividad económica y cooperativas integrales si ejercen diversas ramas de la actividad económica. Dicta normas sobre disolución, liquidación, inspección y vigilancia las cuales quedarán a cargo de la Superintendencia Nacional de Cooperativas. Finalmente, dicta reglas especiales

9 La ley 134 de 1931 no se toma en cuenta en los modelos porque la información recolectada inicia en 1933. Esta ausencia de información por 2 años no permitiría estimar adecuadamente el efecto de esta ley. Para evitar una inadecuada estimación del coeficiente asociado con esta ley, se decide no tomarla en cuenta. No obstante, esta decisión no afecta los modelos ni la validez de los demás coeficientes ya que los errores de los modelos son normales, no correlacionados y homoscedasticos. 
para cooperativas de consumo, ahorro y crédito, producción, trabajo, seguros y cooperativas escolares.

\subsection{Ley 24 de 1981}

Transforma la Superintendencia Nacional de Cooperativas en el Departamento Administrativo Nacional de Cooperativas (DANCOOP), cuyo objetivo y finalidad es: "dirigir y ejecutar la política cooperativista del Estado, colaborar en la planeación económica cooperativa, propiciar el fomento financiero cooperativo, prestar asistencia técnica cooperativa, impartir educación e instrucción cooperativa y ejercer vigilancia y control sobre las sociedades cooperativas, los organismos cooperativos de grado superior, las instituciones auxiliares del cooperativismo, los institutos de financiamiento, educación, investigación y desarrollo cooperativo, los fondos de empleados y las sociedades mutuarias" (Ley 24, 1981:1).

Las principales funciones del DANCOOP serán promover el fomento, la educación y el desarrollo cooperativo, adelantar los estudios de base e investigación necesarios para la formulación de planes y proyectos en pro del sector, ejercer el control y la vigilancia sobre las entidades que cobija su acción, promover la integración económica y social de las entidades cooperativas a nivel regional y nacional, prestar asesoría y asistencia técnica en la constitución y funcionamiento de las entidades sometidas a su control, organizar un sistema de estadística relativo a las entidades a su cargo, propiciar con las instituciones de carácter financiero el apoyo económico para el sector cooperativo, imponer las sanciones previstas en las Leyes cooperativas, en los Decretos y demás disposiciones sobre la materia, congelar los fondos y suspender o clausurar temporal o definitivamente el desarrollo de sus operaciones, suspender temporalmente o cancelar en forma definitiva su personería jurídica, decretar su disolución y ordenar la liquidación de conformidad con la Ley, excluirlas temporal o definitivamente del registro del Departamento Administrativo Nacional de Cooperativas (Ley 24 de 1981).

\subsection{Ley 79 de 1988}

Dota al sector cooperativo de un marco propicio para su desarrollo como parte fundamental de la economía nacional de acuerdo a los siguientes objetivos: "facilitar la aplicación y práctica de la doctrina y los principios del cooperativismo, promover el desarrollo del derecho cooperativo como rama especial del ordenamiento jurídico general, contribuir al fortalecimiento de la solidaridad y la economía social, contribuir al ejercicio y perfeccionamiento de la democracia mediante una activa participación, fortalecer el apoyo del gobierno nacional, departamental y municipal al sector cooperativo, propiciar la participación del sector cooperativo en el diseño y ejecución de los planes y programas de desarrollo económico y social, y propender al fortalecimiento y consolidación de la integración cooperativa en sus diferentes manifestaciones" (Ley 79 de 1988:1). 
En su estructura se presentan las disposiciones generales sectoriales, lineamientos para la constitución y reconocimiento de organizaciones cooperativas, disposiciones sobre los asociados, administración y vigilancia, consideraciones sobre el régimen económico, de trabajo, de fomento y exenciones, definición del tipo de cooperativas y disposiciones especiales aplicables para alguna de ellas, observaciones sobre educación e integración cooperativa, reglas para la fusión, disolución y liquidación. Finalmente, hace referencia a las pre-cooperativas e instituciones auxiliares como organizaciones del sector cooperativo.

\subsection{Ley 454 de 1998}

Esta Ley tiene como objetivo determinar el marco conceptual que regula la economía solidaria. También transforma el Departamento Administrativo Nacional de Cooperativas en el Departamento Nacional de la Economía Solidaria, crea la Superintendencia de la Economía Solidaria y el Fondo de Garantías para las Cooperativas Financieras y de Ahorro y Crédito (FOGACOOP). Dicta normas sobre la actividad financiera de las entidades de naturaleza cooperativa y se expiden otras disposiciones (Ley 454 de 1998).

\section{Evolución del sector cooperativo en Colombia}

La evolución del número y crecimiento de las cooperativas a nivel nacional se muestra en el Gráfico 1 , mientras que el Gráfico 2 lo hace a nivel departamental ${ }^{10}$.

Gráfico. 1. Número y Variación Porcentual de la Cantidad de Cooperativas en Colombia.
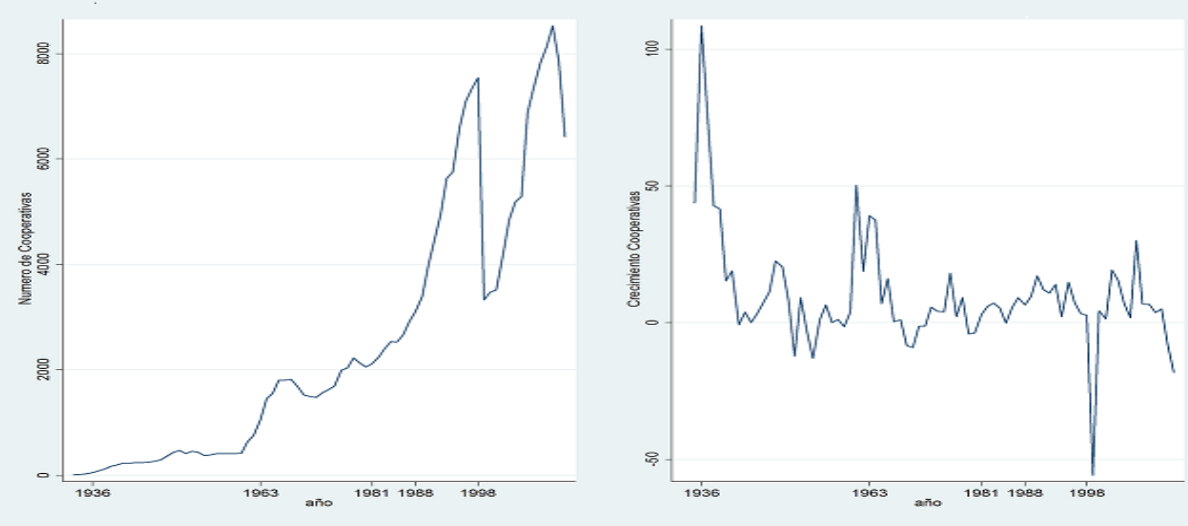

Fuente: Reporte Estadístico de la SuperIntendencia Nacional de Cooperativas, DANCOOP, CONFECOOP y Compilación por los Autores

10 Los departamentos de Antioquia, Valle, Santander y la ciudad de Bogotá son los lugares geográficos con mayor presencia del sector cooperativo en Colombia. 
La evolución del crecimiento promedio de las organizaciones cooperativas evidencia un incremento de $108 \%$ en 1936. Este aumento es el resultado de una conjunción de hecho a saber: primero, el origen en las asociaciones mutuarias que funcionaban a finales del siglo XIX e inicios del XX en los departamentos de Antioquia, Cundinamarca, Boyacá y Nariño (DANCOOP, 1981) ${ }^{11}$; segundo, los esfuerzos de Rafael Uribe Uribe en 1904, Jesús María Fernández en 1915 y Adán Puerto en 1921 para dar a conocer las ventajas de un sistema económico revolucionario denominado cooperativismo; y tercero, como resultado de la crisis económica de 1930 el gobierno Colombiano respaldó la idea cooperativista como conveniente y efectiva.

Entre 1937 - 1960 el crecimiento promedio del sector cooperativo fue de $10.9 \%$. En este periodo se fortalecieron los privilegios y garantías a las sociedades cooperativas consignados en la Ley 134 de 1931 y 128 de 1936. También surgieron organismos cooperativos de orden superior como la Unión Cooperativa de Colombia (UNICOOP) creada en 1948, La Liga Nacional de Cooperativas (LINALCO) constituida en 1951, la Alianza Cooperativa Colombiana en 1954, La unión Cooperativa Nacional de Crédito (UCONAL) fundada en 1959 y la Asociación Colombiana de Cooperativas (ASCOOP) organizada en 1960.

Entre 1961 y 1997 el crecimiento promedio cooperativo fue de 8.61. En esta etapa se inicia el proceso de integración del sector cooperativo. La integración se presenta por la creación y desarrollo de organismos de orden superior que tenían como objetivo la representación, financiamiento y educación del movimiento cooperativo. Al respecto Zabala (2012) se refiere a las razones por las cuales las cooperativas se fortalecen a través de la integración durante las etapas de desarrollo “... el sector cooperativo sólo alcanza esta dimensión cuando sus unidades individuales actúan permanentemente con base en la práctica de la integración y la intercooperación. Pero no sólo es por el hecho de que las cooperativas alcanzan una cierta capacidad numérica o de cobertura territorial o de actividades económicas que les exige algún tipo de coordinación, es la condición misma de encontrarse conviviendo con sectores económicos que le compiten, le ponen barreras que buscan subordinarle, lo que lleva al movimiento a adoptar la forma de federalización empresarial y concentración económica, con el fin de sobrevivir." (Ibid:113). Dentro de los principales organismos cooperativos se encuentra el Instituto Nacional de Financiamiento y Desarrollo Cooperativo (FINANCIACOOP) fundado en 1968. Esta y otras cooperativas de orden superior fueron/son importantes en el fortalecimiento del sector.

Adicionalmente, en la historia del cooperativismo en Colombia es fundamental su contribución al desarrollo de la educación. La Ley 9 de 1971 fomenta los institutos docentes de carácter cooperativo o mutuario, dando lugar a nuevas oportunidades educativas con menores costos, para los sectores populares del país. "Esta ley reglamentada por el Decreto número 2026 de 1973 (octubre 5) fue un medio muy favorable para la promoción de las cooperativas de educación o colegios cooperativos que en los años subsiguientes tuvieron notable desarrollo y

11 Este movimiento mutualista surgió de la necesidad de "dar sepultura a los difuntos desprovistos de recursos económicos, o de la ayuda mutua aplicada a cubrir los riesgos de vejez, enfermedad, muerte, y más adelante, a cubrir los servicios de salud, educación y recreación" (Ibid, p.66). 
representaron una excelente manifestación de actividad conjunta entre el Estado y el Cooperativismo." (Uribe, 2003:32)

Varios autores enmarcan esta etapa de gran participación y desarrollo de las cooperativas de educación en el país, "La tercera característica es la iniciación de un tipo novedoso de cooperativa, producto del trabajo de un grupo de estudiantes de Indesco para optar al grado en Economía Social y Cooperativismo. Se trata de los colegios cooperativos que constituyen una nueva modalidad de empresa cooperativa. Estos colegios llegaron a solucionar un déficit educacional del país, especialmente en las zonas rurales y en los barrios marginados de las grandes ciudades." (Mora, 2012:52)

El periodo comprendido entre 1998-1999 se conoce como la crisis del cooperativismo financiero colombiano. Esta se explica principalmente por el deterioro de las cooperativas del sector financiero debido a la falta de acompañamiento del Estado, la imposición de regulación bancaria comercial, la falta de confianza de los asociados y los malos manejos financieros de los gerentes (Caro, 2013). Esto condujo a una iliquidez y posterior detrimento del sector. Adicionalmente, Fajardo (2003) le atribuye a tres factores el mal desempeño del sector: a) la crisis de la economía colombiana generada por la implementación del modelo económico neoliberal; b) la crisis del sector financiero $\mathrm{y}$, c) el establecimiento de nueva legislación tributarias que afectaron el desarrollo de las cooperativas.

Finalmente, el periodo comprendido entre 2000 a 2014 el número de instituciones cooperativas crece en promedio a tasas de $5.80 \%$ anual.

El Gráfico 2 muestra el crecimiento del sector cooperativo en Antioquia, Valle, Santander y la ciudad de Bogotá ${ }^{12}$. La evolución no es muy diferente a los patrones encontrados a nivel nacional.

12 En estas entidades territoriales se presenta una mayor presencia del sector cooperativo en Colombia. 
Gráfico. 2. Crecimiento Porcentual de Cooperativas en los Principales Departamentos.
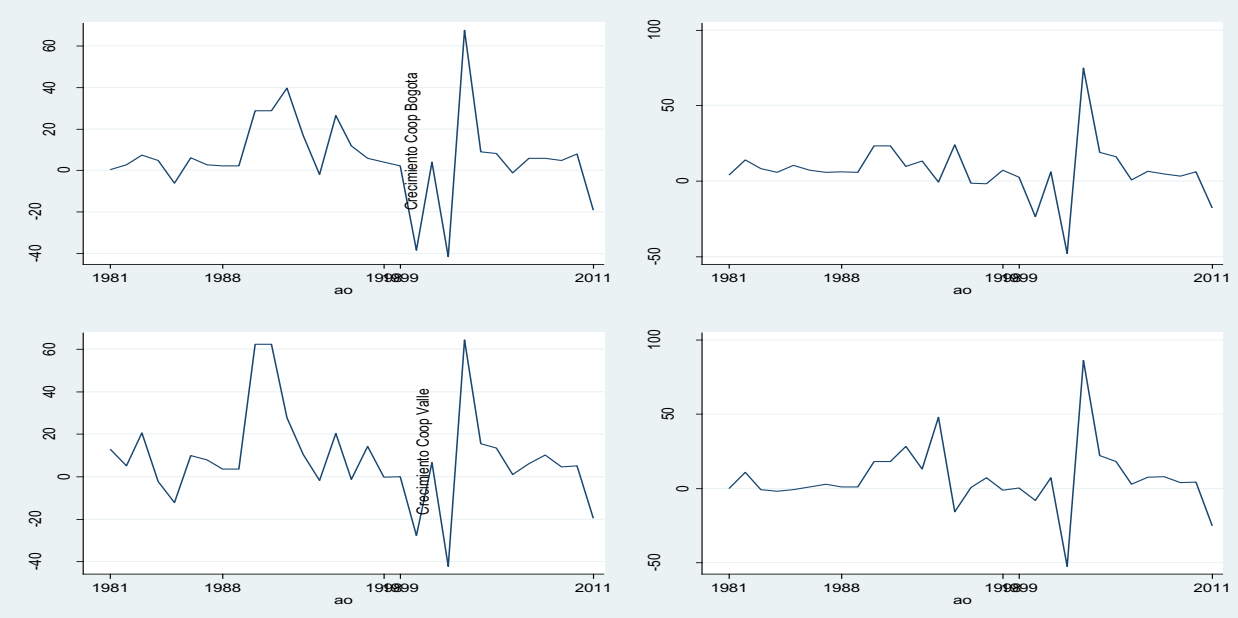

Fuente: Reporte Estadístico de la SuperIntendencia Nacional de Cooperativas, DANCOOP, CONFECOOP y Compilación por los Autores

\section{Metodología}

\subsection{Datos}

La recopilación del número de cooperativas desde 1933 hasta 2014 a nivel nacional y desde 1980 hasta 2014 a nivel departamental se construyó a partir de los estudios sectoriales y anuarios estadísticos de la Superintendencia de Cooperativas, el DANCOOP y los informes anuales que reporta la Confederación de Cooperativas de Colombia (CONFECOOP).

\subsection{Análisis de Intervención}

El análisis de intervención es una técnica econométrica que busca estimar la influencia de ciertos eventos externos en el comportamiento histórico de una serie temporal. Estos eventos externos se denominan intervenciones y están asociados, entre otras acciones, a cambios específicos en la política pública. Según Guerrero (2003), medir el impacto de una intervención es de suma importancia dado que puede ayudar a explicar el comportamiento de la serie y mejorar la estimación de los parámetros y los resultados del modelo.

La técnica de análisis de intervención está basada en la metodología de modelos Auto-regresivos Integrado de Media Móvil (ARIMA) desarrollada por Box y Jenkins en 1976. Bajo esta metodología, una serie temporal que involucre intervenciones externas puede ser expresada como la suma de un modelo ARIMA estacionario invertible y una función que represente los efectos de la intervención. La expresión $T\left(Z_{t}\right)=N_{t}+E_{I, t}$ incorpora a una serie temporal el efecto de una 
intervención. $N_{t}$ representa el modelo temporal estacionario ARIMA $\phi(B) \nabla^{d} N_{t}=\phi_{0}+\theta(B) a_{t}$, y $E_{I, t}$ la función de intervención. Por tanto, para estimar el efecto de una intervención sobre el comportamiento de una serie de tiempo es indispensable estimar un modelo ARIMA estacionario e invertible e identificar la forma funcional de la intervención.

\subsection{Modelos y Estimaciones}

Esta sección tiene como objetivo describir los modelos ARIMA, las variables de control incluidas en cada uno de ellos, y los principales resultados. Se presenta la estructura del modelo de intervención, las pruebas de raíz unitaria, la estimación de los crecimientos promedio dado el marco legislativo cooperativo, y las pruebas de especificación sobre normalidad y correlación de los errores.

\subsection{Modelo}

La ecuación 1 muestra la estructura general del modelo a ser estimado.

$$
Y_{t}=f\left(Y_{t-k} ; I_{i} ; X_{t} ; E_{t-k}\right)
$$

Bajo esta especificación $\mathbf{Y}_{\mathbf{t}}$ y $\mathbf{Y}_{\mathbf{t}-\mathbf{k}}$ son respectivamente el crecimiento anual y los rezagos del crecimiento del sector cooperativo; $\mathbf{I}_{\mathbf{i}}$ es un vector de variables dummies sobre legislación cooperativa que toman el valor de 1 para los años en que la Ley estuvo vigente y 0 si fue derogada (Ley128_1936, Ley 1598_1963, Ley79_1988, Ley24_1981, Ley454_1998); Xt es un vector de variables de control como crecimiento económico y su primer rezago, crecimiento poblacional y su primer rezago ${ }^{13}$, tasa de cambio peso/dólar, variables dummies de cambio de la estructura económica colombiana que toman el valor de 1 después del cambio y cero antes de este (bonanza cafetera (1976-1983); crisis de la deuda (1981-1983); apertura económica (1988 en adelante); enfermedad holandesa por llegada de capitales externos que impulso el sector importador (1993-1998); variables dummies de crisis financieras que toman el valor de 1 durante la misma y cero en otro caso $(1998-2001 ; 2008-2010)$. Finalmente, $\mathrm{E}_{\mathrm{t}-\mathrm{k}}$ son choques aleatorios.

\subsection{Pruebas de Raíz Unitaria}

Antes de estimar los modelos, es necesario garantizar que los datos siguen un proceso estacionario por la metodología ARIMA. El crecimiento cooperativo (nacional y departamental) fluctúa alrededor de una tendencia horizontal, sin grandes movimientos en varianza y correcciones rápidas hacia el promedio. Estos comportamientos son características de series estacionarias. La Tabla. 1 presenta los resultados de las pruebas estadísticas de raíz unitaria (Dickey-Fuller aumentada

13 La inclusión de estas variables de control en los modelos pretenden capturar y filtrar los posibles efectos sobre el crecimiento de las organizaciones cooperativas asociados a incrementos en la población, crecimiento económico o cambios estructurales en la economía como la apertura económica, tasa de cambio y crisis financieras. 
y Phillips Perron). En todos los casos se rechaza la hipótesis nula de raíz unitaria, por lo que se concluye que los crecimientos cooperativos son estacionarios.

Tabla. 1. Prueba de Raíz Unitaria: Nacional y Departamental

\begin{tabular}{|l|c|c|r|l|l|l|}
\hline \multirow{2}{*}{ Nacional/Deptal } & \multicolumn{4}{|c|}{ DICKEY - FULLER } & \multicolumn{3}{l|}{ PHILLIPS - PERRON } \\
\cline { 2 - 7 } & Estadistico & V.Crítico & P-value & Estadistico & V.Crítico & P-value \\
\hline Nacional & -1.948 & -1.69 & 0.029 & -4.225 & -2.908 & 0.000 \\
\hline Antioquia & -5.627 & -1.701 & 0.000 & -5.639 & -2.086 & 0.000 \\
\hline Bogota & -6.658 & -1.701 & 0.000 & -6.662 & -2.086 & 0.000 \\
\hline Santander & -4.62 & -1.701 & 0.000 & -4.639 & -2.086 & 0.000 \\
\hline Valle & -6.386 & -1.701 & 0.000 & -6.367 & -2.086 & 0.000 \\
\hline
\end{tabular}

Fuente: Elaboración propia

Una vez se comprueba que el crecimiento cooperativo es estacionario se procede a estimar los modelos ARIMA con sus respectivas variables de control. Los resultados a nivel nacional se presentan en la Tabla 2 y los departamentales en la Tabla 3.Tabla. 3 Cada una de estas tablas se compone de 3 bloques. En el primero se encuentran los resultados del efecto de la legislación, el segundo muestra los estimadores ARIMA, y el tercero presenta los resultados de las pruebas de especificación sobre residuales (autocorrelación y normalidad) ${ }^{14}$.

Tabla. 2. Resultados Modelos ARIMA a Nivel Nacional

\begin{tabular}{|l|l|l|l|}
\hline VARIABLES & Modelo (1) & Modelo (2) & Modelo (3) \\
\hline \multirow{2}{*}{ Ley128_1936 } & $\mathbf{- 1 . 5 0 9}$ & $\mathbf{- 0 . 9 8 8}$ & $\mathbf{- 0 . 9 1 1}$ \\
\cline { 2 - 4 } & $(271.3)$ & $(286.2)$ & $(367.0)$ \\
\hline \multirow{2}{*}{ Ley1598_1963 } & $\mathbf{8 . 3 4 2}$ & $\mathbf{9 . 2 0 0}$ & $\mathbf{9 . 0 7 8}$ \\
\cline { 2 - 4 } & $(9.635)$ & $(9.085)$ & $(9.102)$ \\
\hline \multirow{2}{*}{ Ley79_1988 } & $\mathbf{5 . 2 1 7}$ & $\mathbf{5 . 2 5 8}$ & $\mathbf{5 . 1 4 1}$ \\
\cline { 2 - 4 } & $(274.7)$ & $(288.7)$ & $(371.2)$ \\
\hline \multirow{2}{*}{ Ley454_1998 } & $\mathbf{- 4 . 0 2 9}$ & $\mathbf{- 3 . 6 7 7}$ & $\mathbf{- 3 . 6 9 9}$ \\
\cline { 2 - 4 } & $(286.4)$ & $(304.5)$ & $(369.9)$ \\
\hline \multirow{2}{*}{ AR } & $\mathbf{- 2 . 0 0 8}$ & $\mathbf{- 0 . 2 2 9}$ & $\mathbf{- 0 . 1 6 8}$ \\
\hline \multirow{2}{*}{ Constant } & $(239.8)$ & $(276.2)$ & $(296.4)$ \\
\cline { 2 - 4 } & $0.982 * * *$ & $0.981 * * *$ & $0.981 * * *$ \\
\cline { 2 - 4 } & $(0.0338)$ & $(0.0350)$ & $(0.0339)$ \\
\cline { 2 - 4 } & -2.364 & -2.339 & -9.484 \\
\hline
\end{tabular}

14 Los coeficientes de las variables de control (apertura económica, población y crecimiento económico) son significativos estadísticamente. Estos no se muestran en la tabla pero pueden ser presentados si así se requiere. 


\begin{tabular}{|l|l|l|l|}
\multirow{3}{*}{ Pormanteau } & $27.49^{*}$ & $31.05^{*}$ & $32.08^{*}$ \\
\cline { 2 - 4 } & {$[0.81]$} & {$[0.65]$} & {$[0.60]$} \\
\hline \multirow{2}{*}{ Jarque Bera } & $4.76^{* *}$ & $7.85^{* * *}$ & $8.47^{* * *}$ \\
\cline { 2 - 4 } & {$[0.09]$} & {$[0.19]$} & {$[0.14]$} \\
\hline
\end{tabular}

El modelo 1 incluye controles asociados a los ciclos del movimiento cooperativo y la apertura económica. El modelo 2 incluye además de los ciclos del movimiento cooperativo, el crecimiento del PIB y su rezago. El modelo 3 incluye, además de los controles del modelo 2, el crecimiento poblacional y su rezago, crisis financieras y variables de tasa de cambio. Todos los modelos son autorregresivos de orden 1. Los errores estándar están en paréntesis y los p-value en paréntesis cuadrados. Niveles de Significancia: ${ }^{* * *} \mathrm{p}<0.01,{ }^{* *} \mathrm{p}<0.05,{ }^{*} \mathrm{p}<0.1$

Fuente: Elaboración propia

Los modelos anteriores ${ }^{15}$ muestran que el crecimiento promedio de las organizaciones cooperativas se estima en $-0.9 \%,-3.6 \%$ y $-0.1 \%$ como consecuencia de las Leyes 128 de 1936, 79 de 1988 y 454 de 1998. "Treinta años después de la primera ley ya existía un desarrollo relativo del movimiento cooperativo colombiano..., sin embargo, débil en su estructura empresarial y sin instituciones bancarias y de seguros de naturaleza cooperativa que apoyaran una mayor consolidación”. (Sarmiento y Guarín, 2002:86)

Por su parte, las Leyes 1598 de 1963 y 24 de 1981 promueven el sector cooperativo con crecimientos promedio de $9 \%$ y $5.1 \%$ respectivamente. No obstante, estos coeficientes no son estadísticamente significativos a ningún nivel de confianza ${ }^{16}$. "Con la reforma aprobada, la creación de la Superintendencia y el apoyo financiero que prestó Financiacoop al movimiento cooperativo colombiano, éste se desarrolló significativamente e incorporó nuevos sujetos de la acción cooperativa, como los profesionales universitarios, pequeños industriales y comerciantes y agricultores medios, todo lo cual contribuyó a un notorio crecimiento empresarial de las cooperativas y a la generación de nuevas instituciones financieras de grado superior e instituciones aseguradoras de naturaleza cooperativa. " (Sarmiento y Guarín, 2002:87)

De manera general, a nivel departamental las Leyes 24 de 1981 y 79 de 1988 ayudan al crecimiento del sector, mientras la Ley 454 de 1998 no lo hace ${ }^{17}$. Adicionalmente, se encuentran magnitudes diferentes en cada región. Por ejemplo, la Ley 24 de 1981 generó un crecimiento de 16.8\% en Antioquia, 12.7\% en Santander, 8.3\% en Bogotá y -4\% en Valle. De la misma forma la Ley 454 de 1998

15 Los modelos estimados son autorregresivos de orden 1, no correlacionados y normales, tal como lo muestra la prueba de Pormanteau y Jarque Bera respectivamente.

16 Los coeficientes de crisis financieras, tasa de cambio y cambios estructurales (excepto apertura económica) no fueron significativos.

17 Las estimaciones se hicieron para los departamentos de Atlántico, Bolívar, Boyacá, Caldas, Caquetá, Cesar, Córdoba, Cundinamarca, Choco, Huila, Guajira, Magdalena, Meta, Nariño, Norte de Santander, Quindío, Caldas, Risaralda, Sucre, Tolima, Norte de Santander. En todos ellos los resultados fueron similares a los encontrados en Antioquia, Bogotá, Santander y Valle, es decir, ningún efecto significativo de la legislación sobre el crecimiento cooperativo. 
tuvo un efecto de $-17.1 \%$ en Antioquia, $-12.4 \%$ en Santander, $-10 \%$ en Bogotá y $6.9 \%$ en Valle. Si bien estos resultados muestran que el efecto de la política es diferencial a nivel regional, su impacto no es estadísticamente significativo.

Tabla. 3. Resultados Modelos ARIMA a Nivel Departamental

\begin{tabular}{|l|l|l|l|l|}
\hline \multirow{2}{*}{ VARIABLES } & Antioquia & Bogotá & Santander & Valle \\
\hline \multirow{3}{*}{ Ley24_1981 } & & & & \\
\hline \multirow{3}{*}{ Ley79_1988 } & $\mathbf{1 6 . 8 8}$ & $\mathbf{8 . 3 1 4}$ & $\mathbf{1 2 . 7 5}$ & $\mathbf{- 4 . 0 2 7}$ \\
\hline & $\mathbf{0 . 0 7 7 0}$ & $(112.5)$ & $(136.9)$ & $(101.5)$ \\
\cline { 2 - 5 } & $(29.59)$ & $\mathbf{2 . 8 9 6}$ & $\mathbf{- 3 . 3 0 5}$ & $\mathbf{1 . 0 9 1}$ \\
\hline \multirow{3}{*}{ AR } & $\mathbf{- 1 7 . 1 3}$ & $\mathbf{- 1 0 . 0 4}$ & $\mathbf{- 1 2 . 4 9}$ & $\mathbf{- 6 . 9 2 3}$ \\
\hline \multirow{2}{*}{ Constant } & $(45.85)$ & $(72.14)$ & $(105.7)$ & $(243.7)$ \\
\cline { 2 - 5 } & $(29.00)$ & $(41.80)$ & $(55.90)$ & $(84.84)$ \\
\hline \multirow{2}{*}{ Pormanteau } & $-0.604^{* * *}$ & $-0.522^{* * *}$ & $-0.365^{* * *}$ & $-0.528^{* * *}$ \\
\cline { 2 - 5 } & $(0.0920)$ & $(0.0805)$ & $(0.112)$ & $(0.0881)$ \\
\hline \multirow{2}{*}{ Jarque Bera } & 47.43 & -12.28 & -77.37 & -11.54 \\
\cline { 2 - 6 } & $(111.3)$ & $(120.0)$ & $(153.7)$ & $(385.2)$ \\
\cline { 2 - 6 } & {$\left[0.47^{* *}\right]$} & {$\left[0.58^{* *}\right]$} & {$\left[0.91^{* *}\right]$} & {$\left[0.35^{* *}\right]$} \\
\cline { 2 - 6 } & 1.698 & 5.4 & 5.5 & 6.65 \\
\hline
\end{tabular}

Los modelos incluyen controles como la apertura económica, el crecimiento del PIB y su rezago, el crecimiento poblacional y su rezago. Errores estándar en paréntesis y p-values en paréntesis cuadrados. Los modelos son autoregresivos de orden 1, no correlacionados y normales. Niveles de significancia: $* * * \mathrm{p}<0.01,{ }^{* *} \mathrm{p}<0.05,{ }^{*} \mathrm{p}<0.1$

Fuente: Elaboración propia

\section{Discusión}

Los resultados encontrados en esta investigación son consistentes con otros trabajos de tipo cualitativo en Colombia. Carlos Uribe expresó que "desde los orígenes del cooperativismo colombiano se ha podido observar, en las respectivas disposiciones legales y reglamentarias, el interés del Estado por favorecer el desarrollo del sistema (...) si la acción no ha sido más eficaz se debe en buena parte a la desproporción entre los objetivos de desarrollo cooperativo señalados en las Leyes y en las declaraciones oficiales, por una parte, y por otra, los recursos suministrados efectivamente por el Estado para el logro de tales objetivos" (Citado por Caro, 2013:225). Uribe (2001) también señala que "en algunos casos la normatividad jurídica no ha sido suficiente para lograr los 
propósitos, y en otros, a resultado contraproducente" (citado por el libro de la Procuraduría General de la Nación, 2011:69).

El estudio adelantado por la Procuraduría General de la Nación muestra que la ley 454 de 1998 "ha tenido una eficacia muy alta en materia de supervisión, pero en materia de fomento y promoción, nuevamente se ha quedado escrita" (Procuraduría General de la Nación, 2011:95). "Nuevamente se demuestra que el Estado no le ha interesado realmente el fomento ni la promoción del cooperativismo y que no se ha ocupado en sus políticas seriamente de estos asuntos, solo se ha interesado por la supervisión" (Ibid:96).

Por su parte, Rymel Serrano (2001) expone que las políticas, estrategias, mecanismos e instrumentos de apoyo, tanto para el sector rural como para el fomento de las empresas asociativas no son adecuadas. ".... Tampoco lo son para generar y consolidar empresas asociativas eficientes capaces de articular estructural y geográficamente el sector de la economía agraria, ni para retener los valores agregados que se generan en la actividad y canalizarlos hacia la economía de la población." (Serrano, 2001:9)

Finalmente, Caro (2013) reconoce que la crisis del cooperativismo se profundizó por la falta de acompañamiento por parte del Estado y la imposición de reglamentación financiera comercial al sector cooperativo financiero.

¿Porque no ha sido más eficaz el marco jurídico cooperativo para lograr el apoyo, desarrollo y fortalecimiento del sector?. Existen varias explicaciones. Primera, ha existido una gran dispersión y falta de coherencia de política; segunda, la regulación ha tenido un enfoque más de supervisión y vigilancia que de fomento y fortalecimiento cooperativo; y tercera, el marco regulatorio no ha tenido en cuenta los efectos negativos potenciales asociados a la estructura de propiedad colectiva que caracterizan a estas organizaciones.

La dispersión de la política se evidencia en la promulgación de cerca de 50 Leyes y Decretos cooperativos desde 1931 hasta 2015. Dar coherencia y continuidad a tan profusa legislación es una tarea compleja que el Gobierno Nacional no ha podido cumplir. "La legislación expedida por el Estado ha sido profusa, es decir, abundante y excesiva en varias materias, en detrimento de la autonomía del sector cooperativo" (Procuraduría General de la Nación, 2011:104)

Como ejemplo de la incoherencia y confusión que ha caracterizado la legislación sobre las cooperativas, Uribe (2003) expone el caso del Decreto Ley 1598 de 1963: “... fue caracterizado por su acertada visión acerca de las características esenciales de las cooperativas y su futuro; sin embargo, dos disposiciones contenidas en el artículo 7 y 14 relacionadas con la condición de los socios trabajadores de las cooperativas y con la obligación de someter a arbitramiento las diferencias o conflictos, dieron lugar a numerosas controversias jurídicas deliberadas posteriormente en ambientes académicos. ” (Ibid: 35)

Por otra parte, Rymel Serrano (1994) confirma que las cooperativas se crean sin tener en cuenta la integralidad en los aspectos de la economía rural debido a que "los conceptos en que se sustenta la organización de las empresas asociativas son por lo general parciales, desintegrados e incompletos. ”(Ibid:8). Esta puede ser una de las razones fundamentales por las cuales se mantiene la dispersión e incoherencia en la legislación colombiana; mientras que, no exista claridad en los conceptos sobre el cooperativismo, difícilmente será acertada la legislación. 
De esta forma " $n o$ ha existido, en las acciones gubernamentales, una politica coherente que integre los distintos esfuerzos que desde diferentes ángulos se han adelantado para el sector. Las actividades de apoyo a la economía solidaria adelantadas por el Estado se han caracterizado en el pasado por una gran dispersión institucional y falta de integración, que ha conducido a la duplicidad de esfuerzos, poca sistematización de experiencias e ineficiencia en las inversiones" (Departamento Nacional de Planeación, 1995:7).

La falta de coherencia también se expresa en la imposición de restricciones a la oferta y la demanda de los servicios cooperativos, en el reconocimiento de nuevas formas asociativas enmarcadas dentro de la economía solidaria, y en el logro solo de objetivos sociales olvidando los económicos. La legislación desea el crecimiento del sector, pero lo limita por las restricciones antes mencionadas.

Con respecto a la oferta de servicios cooperativos, el artículo 11 del capítulo segundo del Decreto 1598 de 1963 establece que "las cooperativas deberán limitar la prestación de servicios al personal asociado" (Congreso de Colombia, Decreto 1598 de 1963: 724), y el artículo 10 de la Ley 79 de 1988 brinda la posibilidad de extender los servicios de la cooperativa al público no asociado siempre y cuando sea en razón del interés social o del bienestar colectivo. La no libertad en la transabilidad de los bienes y servicios cooperativos en la economía de mercado limita la maximización de excedentes y la sostenibilidad de la organización.

Las restricciones a la demanda se asocian con el mercado laboral. El artículo 25 del capítulo tercero del Decreto 1598 de 1963 establece que el porcentaje máximo de trabajadores no asociados a una cooperativa debe ser del $10 \%$. Por su parte, el artículo 59 de la Ley 79 de 1988 establece que "solo en forma excepcional y debidamente justificada, las cooperativas de trabajo asociado podrán vincular trabajadores ocasionales o permanentes no asociados" (Congreso de Colombia, Ley 79 de 1988:9). Los factores de producción no pueden ser elegidos libremente de acuerdo a las necesidades de la organización. Estos son limitados por la regulación.

Por otra parte, el Gobierno Nacional a partir de la Ley 79 de 1988 reconoció a las pre-cooperativas ${ }^{18}$ como formas constitutivas del sector cooperativo. Por otra parte, esta ley incentiva el desarrollo de organizaciones no necesariamente alineadas totalmente con los principios cooperativos (empresas de servicios en la forma de administraciones públicas cooperativas, fondo de empleados, asociaciones mutuales), organizaciones que surgen en detrimento del sector. Esto es así porque al ser entidades sin ánimo de lucro son sustitutas del sector cooperativo. Por ejemplo, la caída registrada en el año 2012 de las cooperativas puede explicarse, parcialmente, por el incremento en el número de pre-cooperativas $\mathrm{y}$ asociaciones.

Desde la perspectiva de metas y objetivos, la Ley reconoce a las cooperativas como organizaciones cuya principal tarea es la satisfacción del bienestar de sus asociados. Pero se olvida que para lograr estos objetivos sociales, deben generar excedentes económicos. Por ejemplo, la Ley 454 de 1998 muestra los principios y

18 Una pre-cooperativa es un grupo de personas que "bajo la orientación y con el concurso de una entidad promotora, se organicen para realizar actividades permitidas a las cooperativas y, que por carecer de capacidad económica, educativa, administrativa, o técnica no están en posibilidad inmediata de organizarse como cooperativas" (Artículo 124, Ley 79 de 1988). 
características del sector desligado del carácter productivo y empresarial de estas organizaciones (Departamento Nacional de Planeación, 2010).

Respecto al enfoque de la política, ésta ha sido más de supervisión y vigilancia que de fomento y fortalecimiento. De las 50 Leyes y Decretos cooperativos, el 6\% se asocian con la formalización del sector, el 31\% con lineamientos para el crecimiento y el $63 \%$ para actividades de monitoreo, vigilancia y control. "No ha habido una política clara del Estado frente al sector que cumpla lo que las Leyes y la propia Constitución de 1991 le ordenan en cuanto a la protección y promoción de las formas asociativas y solidarias de propiedad" (Procuraduría General de la Nación, 2011:103). "El Estado sólo se ha interesado por la supervisión" (Ibid:103)

Sin embargo, Ramírez Baracaldo (2001) reconoce que aunque existe en Colombia legislación sobre la supervisión en cooperativas, ésta no es lo suficientemente explícita. "Una avalancha de normas, como las expedidas recientemente en Colombia, que no constituye un cuerpo jurídico coordinado, puesto que provienen de distintos organismos o instancias gubernamentales, que no se ajusta a la realidad existente, sino que ha sido tomada de otros sistemas, ha creado confusión y dificultad para interpretarlas y para aplicarlas". (Ibid:146)

Finalmente, el marco regulatorio promulga lineamientos que exacerban o alivian los efectos negativos potenciales asociados a la estructura de propiedad colectiva que caracteriza a estas organizaciones. Si bien esta es fundamental para lograr los objetivos sociales y económicos (Álvarez et al, 2000; Fulton y Giannakas, 2001, Guinnane, 2001; Hansen et al, 2002, Eberl, 2004, James y Sykuta, 2004, Spear, 2004; Bertolin et al, 2008; Novkovic, 2008; Othman et al, 2012; Oczkowski, et al, 2013), es ella la que exacerba los conflictos de agencia ${ }^{19}$ (Álvarez et al, 2000; Banerjee et al, 2001; Borgen, 2004; Novkovic, 2009; Othman et al, 2012; Bertolin et al, 2008) y la restricción de capital ${ }^{20}$ (Lerman, et al, 1993; Álvarez et al, 2000; Menard, 2000; Fulton y Giannakas, 2001; Borgen, 2004; Bekkum, 2006; Rebelo, et al, 2008; Mikami, 2010; Othman et al, 2012; Russell, 2014). Estos problemas inciden negativamente en el crecimiento y fortalecimiento de las organizaciones cooperativas.

La restricción de capital se incrementa al sancionar el artículo 5 del capítulo 1 de la Ley 79 de 1988 la cual establece que el "ingreso y retiro de los asociados sea libre y voluntario" (Congreso de Colombia, Ley 79 de 1988:2), que sea necesario garantizar "la igualdad de derechos y obligaciones de sus asociados sin consideración a sus aportes" (Ibid:2), y que "se promueva la integración con otras organizaciones de carácter popular que tengan por fin promover el desarrollo

19 Álvarez et al (2000) identifica los conflictos de agencia como el insuficiente monitoreo (la propiedad colectiva disminuye el incentivo a monitorear a los directivos y asociados), la falta de seguimiento (no hacer seguimiento a las decisiones tomadas en una cooperativa), la influencia de grupos de poder (la heterogeneidad de los intereses de los asociados crea grupos de poder que buscan el beneficio solo de sus integrantes) y la dificultad para tomar decisiones (la heterogeneidad de los intereses de los asociados evita llegar a consensos).

20 La restricción de capital es un fenómeno que se presenta por la dificultad de conseguir los recursos que la organización necesita. Esta restricción es el resultado de los derechos de propiedad vagos o poco definidos (el capital es colectivo y no individual), con horizonte de tiempo heterogéneo (la diversidad en las decisiones de inversión de los asociados no permiten llegar a consensos respecto a los tiempos de la inversión en la cooperativa) e imposibilidad de seleccionar un portafolio óptimo (no es posible decidir en que invertir el capital de la organización). 
integral del hombre" (Ibid:2). A su vez, el artículo 6 del capítulo 1 de la misma Ley prohíbe establecer "con sociedades o personas mercantiles, combinaciones $o$ acuerdos que hagan participar a estas directa o indirectamente, de los beneficios o prerrogativas que las Leyes otorgan a las cooperativas" (Ibid:2).

La restricción de capital también aumenta por la imposibilidad de mercadear su patrimonio (no es posible la venta de acciones), por la dificultad en la consecución de préstamos bancarios de largo plazo (Lerman et al, 1993), por la no enajenabilidad de las participaciones, por la entrada de nuevos socios con los mismos derechos que los antiguos sobre capital y rentabilidad, y por la salida libre y voluntaria de los asociados que conlleva al retiro de sus aportes (Álvarez et al, 2000).

Los problemas de agencia se profundizan por el artículo 6 del capítulo 1 de la Ley 79 de 1988 al sancionar que no está permitido a ninguna cooperativa "establecer restricciones o llevar a cabo prácticas que impliquen discriminaciones sociales, económicas, religiosas o políticas" (Congreso de Colombia, Ley 79 de 1988:2). También por el artículo 21 del capítulo 3 de la misma Ley que expone que los asociados serán "personas naturales legalmente capaces y los menores de edad que hayan cumplido 14 años, las personas jurídicas de derecho público, las personas jurídicas del sector cooperativo y las demás de derecho privado sin ánimo de lucro" (Ibid:4), finalmente, el artículo 39 del capítulo 4 de la Ley 79 establece que "la junta de vigilancia estará integrada por asociados hábiles en número no superior a 3 con sus respectivos suplentes" (Ibid:7).

Las disposiciones anteriores incrementan la heterogeneidad y el número de asociados, que para Banerjee et al (2001), Borgen (2004), Novkovic (2009), Othman et al (2012), Bertolin et al (2008) son factores que disminuyen el éxito de la cooperativa e incrementan los conflictos de agencia. Es importante resaltar que la junta de vigilancia al ser integrada por los propios asociados crea el escenario propicio para la conformación de grupos de poder que busquen beneficio privado en detrimento del bienestar social.

Con respecto a las disposiciones que intentan aliviar los problemas de agencia y restricción de capital, se encuentra el artículo 41 del capítulo 4 de la Ley 79 de 1988 que expone que "la cooperativa tendrá un revisor fiscal con su respectivo suplente quienes deberán ser contadores públicos con matricula vigente" (Ibid:7) y establece en el artículo 43 de la misma Ley que este no podrá desempeñar el cargo de revisor fiscal en la cooperativa del cual sea asociado. Por su parte, el artículo 52 del capítulo 5 establece que la amortización parcial o total de los aportes sociales será procedente "cuando la cooperativa haya alcanzado un grado de desarrollo económico que le permita ejecutar los reintegros y mantener y proyectar los servicios a juicio de la asamblea" (Ibid:8). Si bien este último intenta evitar la salida de capital por el retiro de los asociados, no es claro lo que significa en términos financieros "grado de desarrollo económico". Sería necesario ligar este concepto a límites inferiores de los indicadores de rentabilidad, endeudamiento y liquidez. 


\section{Conclusiones}

La política pública del sector cooperativo en Colombia no ha tenido un efecto estadísticamente significativo sobre el crecimiento del sector. No obstante, se estima que el efecto promedio a nivel nacional fue de $-0.9 \%,-3.6 \%$ y $-0.1 \%$ para las Leyes 128 de 1936, 79 de 1988 y 454 de 1998, y de 9\% y 5.1\% para las Leyes 1598 de 1963 y 24 de 1981.

A nivel regional se encontraron efectos diferenciales, aunque sin lograr significancia estadística. Por ejemplo, la Ley 24 de 1981 generó un crecimiento de $16.8 \%$ en Antioquia, $12.75 \%$ en Santander, $8.3 \%$ en Bogotá (aproximadamente la mitad que en Antioquia) y -4\% en Valle. De la misma forma la Ley 454 de 1998 tuvo un efecto de $-17.13 \%$ en Antioquia, $-12.4 \%$ en Santander, $-10 \%$ en Bogotá y $6.9 \%$ en Valle (cerca de 3 veces menor que en Antioquia).

Como causas principales que explican los resultados anteriores está la gran dispersión y falta de coherencia de la regulación, el enfoque de la política hacia la supervisión y vigilancia que al fomento y fortalecimiento, y la insuficiente comprensión por parte del marco regulatorio de los efectos negativos potenciales de la estructura de propiedad colectiva que caracterizan a estas organizaciones (problemas de agencia y restricción de capital).

\section{Recomendaciones}

El sector cooperativo debe explorar la posibilidad de llevar a cabo innovaciones organizacionales como propuesta de solución a los problemas de agencia y restricción de capital. Estas innovaciones se asocian con la devolución de una fracción de capital invertido si al asociado se retira de la cooperativa, con la emisión de bonos subordinados, con la inclusión de un inversionista externo o listarse en bolsa con acciones preferentes ${ }^{21}$ (Bekkum, 2006). También se debe explorar la posibilidad de la emisión y venta de membresías (Mikami, 2010).

Queda planteado como línea de estudio si estas modificaciones desvirtúan el modelo cooperativo o por el contrario, lo ayudan a fortalecer.

\section{Referencias bibliográficas}

Alvarez, M; Arbesú, P y Fe, C. (2000) Las cooperativas en el marco de la teoría de la agencia. Ciriec-España, Revista de Economía Pública, Social y Cooperativa, № 34, pp. 170-188.

Banerjee, A; Mookherjee, D; Munshi, K y Ray, D. (2001) Inequality, Control Rights, and Rent Seeking: Sugar Cooperatives in Maharashtra. Journal of Political Economy, Vol. 109, No. 1, pp. 138-190.

Barney, J y Hansen, M. (1994) Trustworthiness: Can it be a source of competitive advantage?. Strategic Management Journal, pp. 175-203.

21 Para evitar la pérdida de propiedad de la organización 
Bekkum, V y Bijman, J. (2006) Innovations in Cooperative Ownership: Converted and Hybrid Listed Cooperatives. Business paper presented at the 7 th International Conference on Management in AgriFood Chains and Networks, Ede, The Netherlands, 31 May - 2 June, 2006.

Bertolin, R; Santos, A; Braga, J. y Braga, M. (2008) Assimetria de Informaçăo e Confiança em Interaçőes Cooperativas. RAC, Curitiba, Vol. 12, No 1, pp. 59-81.

Borgen, S (2004) Rethinking incentive problems in cooperative organizations. Journal of Socio-Economics, No. 33, pp. 383-393.

Caro, E. (2013) Cooperativismo y Complejidad. Implementación del cooperativismo financiero en Colombia (1997-2011). Universidad del Rosario.

CONFECOOP (2014) Desempeño Sector Cooperativo Colombiano, 2014. CONFECOOP.

Congreso de Colombia (1936) Ley 128 de 1936. Diario Oficial, número 23331, 11 de noviembre de 1936.

Congreso de Colombia (1963) Decreto 1598 de 1963. Diario Oficial, 17 de julio de 1963.

Congreso de Colombia (1981) Ley 24 de 1981.

Congreso de Colombia (1988) Ley 79 de 1988.

Congreso de Colombia (1998) Ley 454 de 1998.

DANCOOP (1981) Diagnóstico del Cooperativismo Colombiano. Vol. 1, BogotáColombia.

Departamento Nacional de Planeación (1995) Conpes 2823, 15 de noviembre de 1995.

Departamento Nacional de Planeación (2010) Conpes 3639, 1 de febrero de 2010.

Eberl, P. (2004) The Development of Trust and Implications for Organizational Design: a Game- and Attribution-Theoretical Framework. Schmalenbach Business Review, Vol. 56, pp. 258-273.

Fajardo, M. A. (2003) Presencia del cooperativismo en Colombia. Fundación Universitaria de San Gil - UNISANGIL. UniRcoop, Vol. 1, № 2. Colombia.

Fama, E. y Miller, M. (1972) The Theory of Finance. Holt, Rinehart and Winston, New York.

Fulton, M. y Giannakas, K. (2001) Organizational Commitment in a Mixed Oligopoly: Agricultural Cooperatives and Investor-Owned Firms. American Journal of Agricultural Economics, Vol. 83, No. 5, Proceedings Issue, pp. 1258-1265.

Guerrero, V. (2003) Análisis Estadístico de Series de Tiempo Económicas. Thomson.

Guinnane, T. (2001) El "amigo y consejero": gestión, auditoría y confianza en las cooperativas de crédito alemanas (1889-1914). AREAS Revista de Ciencias Sociales, № 21, pp. 39-61.

Hansen, M; Morrow, J. y Batista, J. (2002) The Impact of Trust on Cooperative Membership Retention, Performance, an Satisfaction: an Exploratory Study. International Food and Agribusiness, $\mathrm{N}^{\circ}$ 5, pp. 41-59.

James, H. y Sykuta, M. (2004) Farmer Trust in Agricultural Cooperatives: Evidence from Missouri Corn and Soybean Producers. Paper prepared for presentation at the 2004 meetings of the American Agricultural Economics Association, in Denver, Colorado, August 1-4, 2004.

Jensen, M. y Meckling, W. (1976) Theory of the firm: Managerial Behaviour, Agency Costs and Ownership Structure. Journal of Financial Economics, Vol. 5, No. 4, pp. 305-360.

Lerman, Z. y Parliament, C. (1993) Financing Growth in Agricultural Cooperatives. Review of Agricultural Economics, Vol. 15, No. 3, pp. 431-441.

Menard, C. (2000) Institutions, Contracts, and Organizations: Perspective fron New Institutional Economics. 
Mikami, K. (2010) Capital procurement of a consumer cooperative: Role of the membership market. Economic Systems 34, P.P.178-197.

Mora, M. y Pardo, L. (2014) La Historia del Cooperativismo en Colombia: Hitos y Periodos. Cooperativismo \& Desarrollo, Vol 22, № 104.

Myers, S.C. (1977) Determinants of Corporate Borrowing. Journal of Financial Economics, Vol. 5, No. 2, pp. 147-175.

Myers, S.C. (1984) The capital structure puzzle. Journal of Finance, No. 39, pp. 575-592.

Myers, S.C. y Majluf, N. (1984) Corporate investment decisions when firms have information that investors don't have. Journal of Financial Economics, Vol. 13, pp. 187221.

Novkovic, S. (2008) Defining the Co-operative Difference. The Journal of SocioEconomics, No. 37, pp. 2168-2177.

Oczkowski, E; Krivokapic-Skoko, B. y Plummer, K. (2013) The meaning, importance and practice of the co-operative principles: Qualitative evidence from the Australian cooperative sector. Journal of Co-operative Organization and Management, pp. 54-63.

Othman, A; Kari, F; Jani, R. y Hamdan, R. (2012) Factors Influencing Cooperative Membership and Share Increment: An Application of the Logistic Regression Analysis in the Malaysian Cooperatives. World Review of Business Research, Vol. 2, No. 5, pp. $24-35$.

Procuraduría General de la Nación (2011) Políticas Públicas y Cooperativismo en Colombia. Instituto de Estudios del Ministerio Público. IEMP ediciones.

Ramírez, B. (2001) La Supervisión y el Control de las Cooperativas. ARFO editores.

Razeto, L. (2010) ¿Qué es la Economía Solidaria?. Papeles de Relaciones Ecosociales y Cambio Global, $\mathrm{N}^{\mathrm{o}} 110$, pp. 47-52.

Rebelo, J; Caldas, J. y Matulich, S. (2008) Manager Power, Member Behavior and Capital Structure: Portuguese Douro Wine Cooperatives. Agricultural Economics Review, Vol. 9, No 2, pp. 5-15.

Russell, L. y Briggeman, B. (2014) The Effect of Taxes on Capital Structure in Farm Supply and Marketing Cooperatives. SAEA 2014 Annual Meetings Selected Paper Presentation January 16.

Sarmiento, A. y Guarin, B. (2002) Aspectos Legales de la Gestión Cooperativa. Pontificia Universidad Javieriana. Colombia.

Serrano, R. (1994) Planificación Participativa. Tacti-k Creativa.

Spear, R. (2004) El gobierno democrático en las organizaciones cooperativas. CiriecEspaña, Revista de Economía Pública, Social y Cooperativa, № 48, pp. 11-30.

Uribe, C. (2003) Derecho Cooperativo. Fondo Nacional Universitario. Bogotá.

Zabala, H. (2012) La Integración desde la Práctica: Criterios y Denominadores Comunes para la cooperación Sectorial. Cooperativismo \& Desarrollo, Vol. 20, № 101. 